\title{
DETERMINACIÓN ESPECTROFOTOMÉTRICA DE ESPECIES INORGÁNICAS ARSENICALES (III) Y (V) EN AGUA USANDO EL MÉTODO DEL AZUL DE MOLIBDENO.
}

Spectrophotometric determination of inorganic arsenic (III) and (V) in water using the molybdenum blue method

\author{
Mario César CORRAL-ESCÁRCEGA ${ }^{1}$, Eduardo RODRÍGUEZ DE SAN MIGUEL-GUERRERO², \\ Josefina DE GYVES MARCINIAK ${ }^{2}$, Guillermo GONZÁLEZ SÁNCHEZ ${ }^{3}$ y \\ María de Lourdes BALLINAS-CASARRUBIAS ${ }^{1 *}$
}

${ }^{1}$ Facultad de Ciencias Químicas, Universidad Autónoma de Chihuahua, Circuito Universitario, Campus II. C.P. 31124. Chihuahua, Chihuahua, México

${ }^{2}$ Facultad de Química, Universidad Nacional Autónoma de México, Ciudad Universitaria, Coyoacán, Ciudad de México C.P. 04510 México

${ }^{3}$ Centro de Investigación en Materiales Avanzados, Miguel de Cervantes 120, Complejo Industrial Chihuahua Chihuahua, Chihuahua. C.P. 31136. México

*Autora para correspondencia: lourdes.ballinas@gmail.com

(Recibido febrero 2018, aceptado abril 2019)

Palabras clave: arsénico, especiación, análisis en agua

\section{RESUMEN}

La contaminación de las aguas superficiales y subterráneas por arsénico ha sido reportada en muchos países en desarrollo. Por esta razón es necesario cuantificarlo utilizando herramientas sencillas y económicas. El principal objetivo de este estudio fue desarrollar un método colorimétrico para la especiación de As-inorgánico en presencia de fósforo $(\mathrm{P}(\mathrm{V}))$, en agua. El método se sustentó en la generación de un heteropoliácido de color azul, en medio ácido, por la reacción del molibdato con el arsenato, pero no con el arsenito. Para la cuantificación de As total se utilizó el yodato de potasio $\left(\mathrm{KIO}_{3}\right)$ para la oxidación cuantitativa del arsénico trivalente (As (III)). Posteriormente, por diferencia del As total y el As (V) se determinó el As (III), restando también la concentración de $\mathrm{P}(\mathrm{V})$. Con el uso de la cisteína se cuantificó el $\mathrm{P}(\mathrm{V})$ mediante la reducción selectiva del As (V). Este método permitió determinar especies arsenicales inorgánicas en agua salobre incluso a nivel de concentraciones de ultra traza $(\mu \mathrm{g} / \mathrm{L})$, obteniendo los siguientes parámetros de calidad analítica para As $(\mathrm{V})$ : límite de detección de $6 \mu \mathrm{g} / \mathrm{L}$, límite de cuantificación de $18 \mu \mathrm{g} / \mathrm{L}$, linealidad de 18 a $1600 \mu \mathrm{g} / \mathrm{L} ; 96.9 \%$ de recuperación y 1.24 $\%$ de precisión. Los resultados fueron obtenidos en un menor tiempo sin la utilización de grandes equipos, a bajo costo y comparables con técnicas analíticas instrumentales como la espectrometría de absorción atómica con horno de grafito.

Key words: arsenic, speciation, water analysis

\begin{abstract}
Arsenic surface water and groundwater pollution is a reported issue in many developing countries, thus it is of great concern to quantify this species by using economic techniques easy to handle. In this work, a spectrophotometric method for the quantification
\end{abstract}


and speciation of arsenic in the presence of phosphorous in water was developed. It is based on the generation of a colored complex (heteropolyacid) in acid media, by arsenate reaction with molybdate. For total arsenic determination, potassium iodate was used for the quantitative oxidation of trivalent arsenic. Trivalent arsenic was determined from the difference between the total arsenic quantified and the arsenate. Phosphorous (V) was quantified by using L-cysteine, by the selective reduction of pentavalent arsenic. The developed method allowed to quantify arsenic at ultra-trace levels $(\mu \mathrm{g} / \mathrm{L})$, presenting the following figures of merit of the analysis: Detection limit $6 \mu \mathrm{g} / \mathrm{L}$, quantification limit $18 \mu \mathrm{g} / \mathrm{L}$, linearity from 18 to $1600 \mu \mathrm{g} / \mathrm{L} ; 96.9 \%$ of recovery and $1.24 \%$ of precision. The results were comparable to those obtained by atomic absorption spectrometry with graphite furnace.

\section{INTRODUCCIÓN}

El arsénico (As) es un metaloide que se encuentra en la corteza terrestre. Por tal razón es común encontrarlo en cuerpos de agua y en el suelo. La contaminación del agua potable por el As es un problema a nivel mundial y cada vez más personas son afectadas por su causa, principalmente a través del agua potable (Dhar et al. 2004). El As ha tomado importancia debido a los efectos en la salud de los humanos (Ma et al. 2014), tales como el engrosamiento y decoloración de la piel, dolor de estómago, náuseas, vómitos, diarrea, entumecimiento en manos y pies, parálisis parcial, ceguera, cáncer de piel, pulmón y vejiga (Das y Sarkar 2016). La concentración de As en el agua puede variar en más de cuatro órdenes de magnitud (0.01-100 ppm). Este metaloide se encuentra en aguas subterráneas bajo condiciones naturales, como resultado de las interacciones agua-suelo y la movilización y acumulación debido a condiciones físicas y geoquímicas favorables, como la oxidación de la arsenopirita, un mineral que lo contiene (Smedley y Kinniburgh 2002). De esta forma, existen acuíferos con concentraciones de As superiores a $50 \mu \mathrm{g} / \mathrm{L}$ en algunos países como es el caso de Argentina, Bangladés, Chile, China, Hungría, India (Bengala Occidental), México, Rumania, Taiwan, Vietnam y suroeste de los Estados Unidos de América, entre otros, lo que supone un grave problema para la salud humana, ya que en muchos casos el agua subterránea se emplea para el abastecimiento de la población (Nordstrom 2002, Smedley y Kinniburgh 2002). La Organización Mundial de la Salud (OMS) recomienda como límite aceptable una concentración de $0.01 \mathrm{mg} / \mathrm{L}$ de As en agua potable (Kiso et al. 2015, Das et al. 2016) por lo que para estas regiones es un tema urgente el monitoreo de los niveles de contaminación de As en agua (Dhar et al. 2004).
El análisis del As (V) puede realizarse mediante el método del azul de molibdeno (MB), con el cual también se realiza el análisis de $\mathrm{P}(\mathrm{V})$ inorgánico y silicato (Kiso et al. 2015, Dhar et al. 2004). El método del MB se describe a detalle por la Asociación Oficial de Químicos Analíticos (AOAC) y es el sugerido para la determinación de As en alimentos, plantas y agua; esto debido a su facilidad de implementación y costo, comparado con técnicas como la espectrometría de absorción atómica. Por otra parte, la Agencia Estadounidense de Protección Ambiental (USEPA) ha revisado el estado del arte para la medición y cuantificación del As en el ambiente. Los métodos aprobados incluyen la espectrometría de masas con plasma acoplado por inducción (ICP-MS), espectrometría de emisión atómica con plasma acoplado por inducción (ICP-AES), absorción atómica con horno de grafito (GFAAS) y espectrometría de absorción atómica con generador de hidruros (HGAAS), cuyos límites de detección (LD) comprenden un intervalo de 0.5 a $50 \mathrm{mg} / \mathrm{L}$ (Martinez et al. 2005). Si bien todos estos métodos cumplen con la mayoría de los parámetros de calidad analítica necesaria para la determinación del elemento a nivel de ultra trazas, los instrumentos utilizados son voluminosos y con altos costos de operación y mantenimiento (Ma et al. 2014, Dhillon et al. 2015). Por tanto, no son convenientes para un monitoreo continuo. En consecuencia, se necesita un método de detección rápido y simple para el análisis de As en el agua potable y de efluentes de plantas de eliminación de As. Una herramienta de monitoreo rápido puede ser útil, incluso, para la investigación de campo, adicional a las técnicas comerciales existentes (Kiso et al. 2015, Dhillon et al. 2015).

En el caso particular de aguas subterráneas, el As se encuentra predominantemente como As (III) y As (V), con cantidades menores de metil y dimetil As. Las especies más tóxicas son las inorgánicas, y el As (III) es documentado como más tóxico que el As (V) 
(Martinez y Gásquez 2005). Su movilidad y toxicidad depende de su forma y estado de oxidación, razón por la cual no sólo es necesaria la determinación total, sino también realizar su especiación, de modo que pueda conocerse la concentración de cada una de las especies presentes. Un reto importante estriba en la cuantificación de cada especie. Existen pocos métodos instrumentales reportados para determinar As (V) y As (III) tomando en cuenta el P (V) como interferente en soluciones acuosas (Cordero et al. 2009, Ma et al. 2014). El método del azul de molibdeno (MB) se basa en la generación de complejos coloridos en medio ácido. Esto se debe a la reacción entre el molibdato ácido y el As (V) y/o el P (V), para formar así el correspondiente molibdo heteropoliácido de coloración amarilla, el cual es posteriormente reducido por el cloruro estañoso a un compuesto de coloración azul. Esta reacción ocurre con el arsenato y es nula con el arsenito. Su principal interferencia es el P(V) presente en la disolución (Charlot 1961, Nagul et al. 2015).

$\mathrm{El} \mathrm{pH}$ es un factor importante a controlar en la cuantificación utilizando el MB, pues de la acidez en el medio de reacción dependerá la formación de las especies coloridas. Una acidez de $160.81 \mathrm{mmol} / \mathrm{L}$ proporciona el medio para la formación de especies coloridas y su relación será directamente proporcional a la concentración de las mismas. Tsang et al. (2007) describieron la estabilidad de los complejos de arsenomolibdato y fosfomolibdato formados en presencia de $\mathrm{H}_{2} \mathrm{SO}_{4}$. Estos autores encontraron que en presencia de $\mathrm{H}_{2} \mathrm{SO}_{4}$ disminuía la tasa de formación del complejo y, por ende, el desarrollo del color, pero también aumentaba la estabilidad del complejo cromóforo al disminuir su velocidad de polimerización.

El objetivo del presente estudio fue desarrollar un método espectrofotométrico para la determinación en agua de As (III) y As (V) en presencia de P (V), mediante la técnica del MB. El método emplea agentes oxidantes y reductores para posteriormente efectuar la reacción entre el molibdato ácido y el As (V) y/o el P (V). Se forma el molibdo heteropoliácido de coloración amarilla para posteriormente ser reducido por el cloruro estañoso a un compuesto de coloración azul (Charlot 1961, Nagul et al. 2015) y para conocer la concentración de cada especie se utiliza la espectrometría UV-Vis.

\section{MATERIALES Y MÉTODOS}

\section{Materiales}

La solución estándar de As (V) a $1000 \mathrm{mg} / \mathrm{L}$ fue preparada pesando $424.92 \mathrm{mg}$ de la sal de arsenato
$\left(\mathrm{Na}_{2} \mathrm{HAsO}_{4} \cdot \mathrm{H}_{2} \mathrm{O}, 98 \%\right.$ de pureza, grado ACS, Sigma-Aldrich) disueltos y aforados a $100 \mathrm{~mL}$ con $\mathrm{HNO}_{3}$ al 2\% v/v. Para el As (III) se pesaron $17.33 \mathrm{mg}$ de la sal de arsenito $\left(\mathrm{NaAsO}_{3}, 90 \%\right.$ de pureza, Sigma-Aldrich) aforando a $10 \mathrm{~mL}$, para dar lugar a una solución 0.01 molar $(\mathrm{M})$. La solución estándar de $1000 \mathrm{mg} / \mathrm{L}$ de $\mathrm{P}(\mathrm{V})$ se preparó a partir de la sal $\left(\mathrm{NaH}_{2} \mathrm{PO}_{4} \cdot \mathrm{H}_{2} \mathrm{O}\right.$ y $\mathrm{NaH}_{2} \mathrm{PO}_{4} \cdot 2 \mathrm{H}_{2} \mathrm{O}, 99 \%$ de pureza, Merck). El reactivo de molibdato de amonio con ácido sulfúrico se preparó pesando $526.31 \mathrm{mg}$ de la sal de molibdato de amonio $\left(\mathrm{H}_{24} \mathrm{Mo}_{7} \mathrm{~N}_{6} \mathrm{O}_{24} \bullet 4 \mathrm{H}_{2} \mathrm{O}\right.$, al 81.0-83.0 \% base $\mathrm{MoO}_{3}$, J. T. Baker) añadiendo $4.505 \mathrm{~mL}$ de ácido sulfúrico $\left(\mathrm{H}_{2} \mathrm{SO}_{4}, 95 \%\right.$ de pureza, J.T. Baker) para hacer la mezcla y aforarlos con 10 $\mathrm{mL}$ con agua. Esta solución tuvo una concentración de $0.04 \mathrm{M}$ en molibdeno y $8.0 \mathrm{M}$ en ácido sulfúrico. Se utilizó agua desionizada para la preparación de todas las disoluciones.

Para la determinación de As (V), (III) y P (V) se preparó la solución estándar reductora pesando $420.2 \mathrm{mg}$ de la sal de cloruro estañoso $\left(\mathrm{SnCl}_{2}\right.$, con pureza del $98 \%$, Sigma-Aldrich) y adicionando $1.25 \mathrm{~mL}$ de ácido clorhídrico al 37.2\% de pureza. A partir de la solución estándar reductora de $\mathrm{SnCl}_{2}$ se preparó la solución de trabajo con una concentración de $0.84 \mathrm{M}$. Para la cuantificación de As (V) y (III) en presencia de $\mathrm{P}(\mathrm{V})$ se prepararon las soluciones de cisteína y yodato de potasio $0.007 \mathrm{M}$. Para el reactivo de cisteína se pesaron $10 \mathrm{mg}$ de la sal $\left(\mathrm{C}_{3} \mathrm{H}_{7} \mathrm{NO}_{2} \mathrm{~S} \cdot \mathrm{HCl}\right.$, $99 \%$ de pureza, Sigma-Aldrich), mientras que el yodato de potasio se preparó pesando $15.04 \mathrm{mg}$ de la sal $\left(\mathrm{KIO}_{3}, 99.7 \%\right.$ de pureza, J.T. Baker) en un volumen de $10 \mathrm{~mL}$.

\section{Análisis de As (V)}

Las soluciones se realizaron en tubos Hach de $13 \times 100 \mathrm{~mm}$, adicionando $100 \mu \mathrm{L}$ de la solución $0.04 \mathrm{M}$ de $\mathrm{H}_{24} \mathrm{Mo}_{7} \mathrm{~N}_{6} \mathrm{O}_{24} \bullet 4 \mathrm{H}_{2} \mathrm{O}$ en $\mathrm{H}_{2} \mathrm{SO}_{4} 8.0 \mathrm{M}$, seguido de la adición del As y la cantidad necesaria de agua para un volumen final de $5 \mathrm{~mL}$. Posteriormente, se adicionaron $200 \mu \mathrm{L}$ de la solución de cloruro estañoso $(0.006 \mathrm{M})$ para el desarrollo del color. Cada tubo se sometió a calentamiento en baño María a $55^{\circ} \mathrm{C}$ por $10 \mathrm{~min}$. Una vez transcurrido el tiempo en el baño María se procedió a enfriar cada estándar a temperatura ambiente por $10 \mathrm{~min}$, para después realizar las lecturas a $840 \mathrm{~nm}$ con celdas de cuarzo en un espectrofotómetro Perkin Elmer Lambda 25.

Para la especiación de As en presencia de $\mathrm{P}(\mathrm{V})$ por el MB, se utilizó la cisteína y el yodato de potasio por separado. Se utilizaron estándares de As (III), As (V) y P (V) para dar las concentraciones finales de la curva de calibración en el rango de 8 a 
$1600 \mu \mathrm{g} / \mathrm{L}$. En primer término, se adicionaron 100 $\mu \mathrm{L}$ de la solución $0.04 \mathrm{M}$ de $\mathrm{H}_{24} \mathrm{Mo}_{7} \mathrm{~N}_{6} \mathrm{O}_{24} \bullet 4 \mathrm{H}_{2} \mathrm{O}$ en medio de $\mathrm{H}_{2} \mathrm{SO}_{4} 8.0 \mathrm{M}$ en dos diferentes tubos Hach en los cuales se encontraban aproximadamente $3 \mathrm{~mL}$ del estándar compuesto. En un tubo aparte se adicionaron el estándar compuesto de As (V), P (V) y As (III) en diferentes cantidades para dar las concentraciones finales de la curva de calibración y $50 \mu \mathrm{L}$ del reactivo de cisteína y se dejaron reposar por 10 min para después agregar $100 \mu \mathrm{L}$ del reactivo de molibdato de amonio/ ácido sulfúrico. A uno de los dos primeros tubos descritos se le agregaron $10 \mu \mathrm{L}$ del reactivo de yodato de potasio. A los tres tubos se les agregó la cantidad de agua necesaria para aforar a $5 \mathrm{~mL}$ en un tubo Hach y, por último, se adicionaron $200 \mu \mathrm{L}$ de la solución de trabajo de $\mathrm{SnCl}_{2}$. Los tubos se taparon herméticamente y se introdujeron en baño María a $52{ }^{\circ} \mathrm{C}$ por $10 \mathrm{~min}$. Transcurrido el tiempo de calentamiento se dejaron enfriar a temperatura ambiente por $10 \mathrm{~min}$ para su posterior lectura a una longitud de onda de $840 \mathrm{~nm}$ en el espectrofotómetro y utilizando celdas de cuarzo.

Para el cálculo de la concentración de $\mathrm{P}(\mathrm{V})$, de la mezcla de As (V), (III) y P (V), se requiere la reducción selectiva del arsenato a arsenito mediante el uso de la cisteína (Fig. 1). Para cuantificar el As total se utilizó el yodato de potasio mediante la oxidación completa del As (III) a As (V) (Fig. 1). Posteriormente, por diferencia del arsénico total menos el As (V) se determinó el As (III), restando también la concentración de fósforo.

Se estudió el efecto de la concentración de $\mathrm{KIO}_{3}$ sobre la señal de As (V) y P (V) a cinco diferentes concentraciones en el intervalo comprendido entre $0.02 \mathrm{mM}$ y $0.14 \mathrm{mM}$.

En la figura 1 se muestra la metodología para la cuantificación y especiación de As (III y V) y P (V) en muestras de agua potable.

Las figuras de mérito del análisis para As (V) fueron obtenidas una vez establecida la técnica del MB utilizando un estándar certificado del Centro Nacional de Metrología (CENAM), además de una muestra real de agua potable. Se determinó la sensibilidad, rango lineal, límite de detección (LD), límite de cuantificación (LC), precisión en términos de coeficiente de variación, \% de recuperación y exactitud mediante la comparación de medias con respecto a la media del estándar certificado DMR-312b de $997 \pm 12 \mathrm{mg} / \mathrm{L}$ de As adquirido del CENAM. Para el análisis se preparó un estándar de $800 \mu \mathrm{g} / \mathrm{L}$ mediante la adecuada dilución del estándar certificado. El LD fue definido como tres veces la desviación estándar (d.e.) del blanco de reactivos.

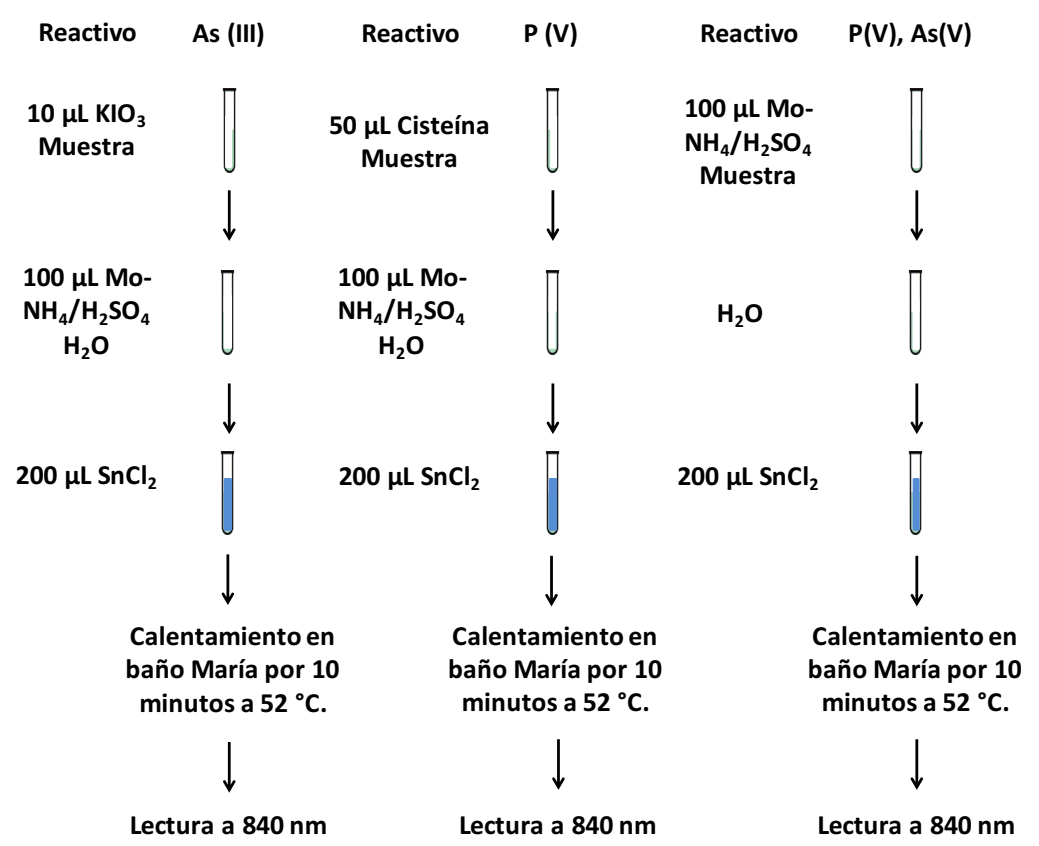

Fig. 1. Proceso de especiación para As (III, V) y cuantificación de interferentes $\mathrm{P}(\mathrm{V})$ 


\section{RESULTADOS Y DISCUSIÓN}

Determinación de As (III) y As (V). Efecto de la concentración de la cisteína y el yodato de potasio.

$\mathrm{El} \mathrm{KIO}_{3}$ es una especie oxidante que tiene influencia sobre la formación del complejo que presenta el color y, por lo tanto, en el predominio de especies químicas. A concentraciones de $0.02 \mathrm{mmol} / \mathrm{L}$, como se observa en la figura 2, la absorción máxima se registra en 710 y $840 \mathrm{~nm}$ para las especies químicas de $\mathrm{P}(\mathrm{V})$ y As (V), respectivamente. El predominio de las especies químicas coloridas se ve favorecido a bajas concentraciones de $\mathrm{KIO}_{3}$; la absorción aumenta conforme disminuye la concentración final de $\mathrm{KIO}_{3}$ en la mezcla de reacción en ambos analitos. A concentraciones de $0.14 \mathrm{mmol} / \mathrm{L}$, el $\mathrm{KIO}_{3}$ puede inhibir la formación de las especies químicas coloridas de As y P. Este efecto puede ser debido a la inactivación de las especies redox participantes, tales como el cloruro estañoso utilizado como agente reductor (Tsang et al. 2007).

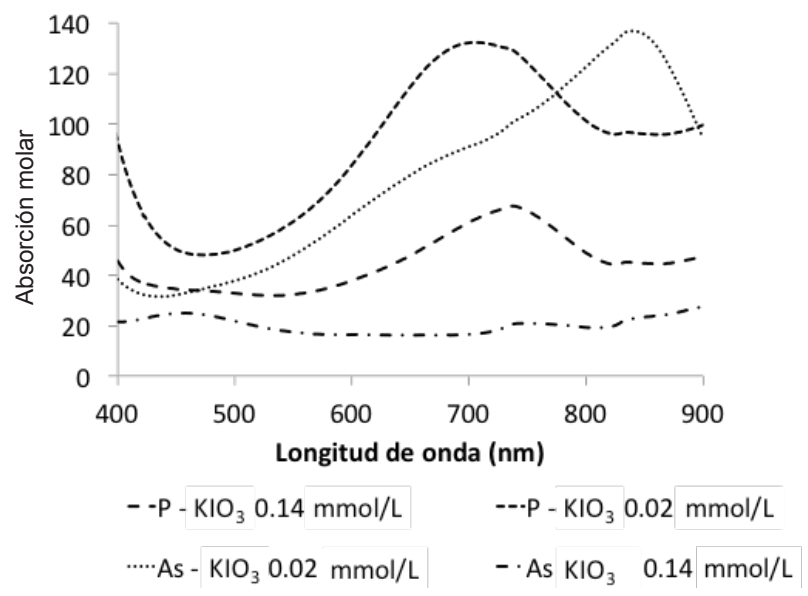

Fig. 2. Absorción molar de las especies químicas (P y As) en función de la concentración de yodato de potasio

En otros trabajos se ha reportado la utilización de diferentes reactivos para la reducción de As (V) a As (III), así como también para la oxidación de la especie de polioxometalato (Ma et al. 2014). En el presente método se utilizó cisteína como agente reductor en medio ácido y se demostró que no se presenta ninguna interferencia en la cuantificación (Fig. 3a).

El mismo efecto se observa en la absorbancia, la cual decrece conforme aumenta la concentración de $\mathrm{KIO}_{3}$ en ambos analitos (Fig. 3b).

Una vez realizados estos estudios se calcularon, bajo las condiciones optimizadas de agentes redox,
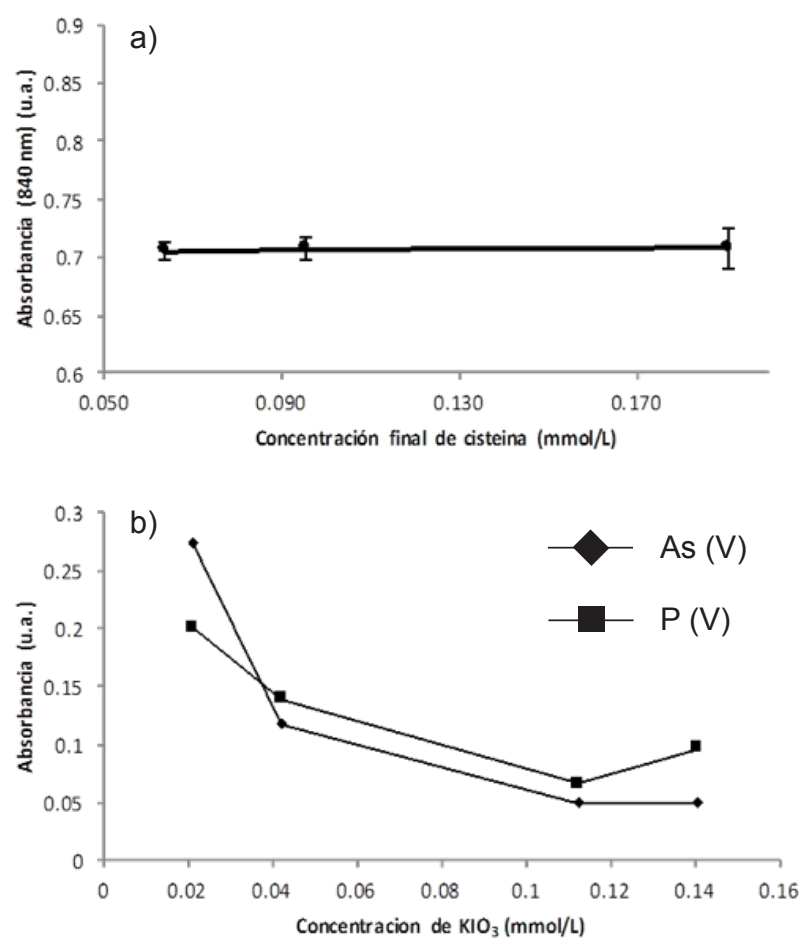

Fig. 3. Efecto de la cisteína (a) y el yodato de potasio (b) en la absorbancia del complejo colorido de molibdeno

los diferentes parámetros de calidad analítica de las tres especies, fósforo pentavalente, arsénico (V) y arsénico (III) (Cuadro I).

Se observó diferencia en el límite de detección y la sensibilidad para las especies arsenicales, obteniendo una mayor sensibilidad para el arsenito, como se muestra en la figura 4 y en el Cuadro I. Esto es resultado de la variación en la matriz de reacción por la adición de $\mathrm{KIO}_{3}$ para la determinación de arsenito, por lo cual el análisis tiene una estrecha dependencia con la naturaleza de la matriz.

Como parte de la validación se realizó la cuantificación de As total por triplicado en una muestra de agua para consumo, comparando el resultado con el obtenido por el método de generador de hidruros (GHAAS); ambos métodos muestran resultados comparables (Cuadro II) al $95 \%$ de confianza acorde con el resultado de la prueba de $t$ pareada para ambos conjuntos de valores (valor $\mathrm{p}=0.7258$ ).

Tsang et al. (2007) reportaron la determinación de As (V) en presencia de $\mathrm{H}_{3} \mathrm{PO}_{4}$ utilizando $\mathrm{S}_{2} \mathrm{O}_{4}{ }^{-2}$ para la reducción del arsenato, pero el método no fue evaluado con muestras de agua reales. Estos investigadores determinaron $0.7 \mu \mathrm{g} / \mathrm{L}$ como LD para As (V) y un rango lineal que comprendió de 0 a $375 \mu \mathrm{g} / \mathrm{L}$. Además, compararon el desarrollo de 
CUADRO I. FIGURAS DE MÉRITO DE ANÁLISIS PARA LA CUANTIFICACIÓN DE As (V), As (III) Y P (V)

\begin{tabular}{lccccrrr}
\hline Analito & $\begin{array}{c}\text { Sensibilidad } \\
(\mu \mathrm{g} / \mathrm{L})\end{array}$ & Intercepto & $\begin{array}{c}\text { Rango lineal } \\
(\mu \mathrm{g} / \mathrm{L})\end{array}$ & $\mathrm{R}^{2}$ & $\begin{array}{c}\mathrm{LD} \\
(\mu \mathrm{g} / \mathrm{L})\end{array}$ & $\begin{array}{c}\% \\
\text { Recuperación }\end{array}$ & $\begin{array}{c}\% \\
\mathrm{CV}\end{array}$ \\
\hline As $(\mathrm{V})$ & 0.0002 & 0.0028 & $8-1600$ & 0.9980 & 6.0 & 97.0 & 1.2 \\
$\mathrm{P}(\mathrm{V})$ & 0.0007 & 0.0283 & $8-800$ & 0.9964 & 2.5 & 110.7 & 5.6 \\
As(III) & 0.0003 & 0.0000 & $8-1600$ & 0.9990 & 15.7 & 84.8 & 10.7 \\
\hline
\end{tabular}

*Los datos de recuperación y \% CV se calcularon con el estándar de $100 \mu \mathrm{g} / \mathrm{L}$ con una n $=5$

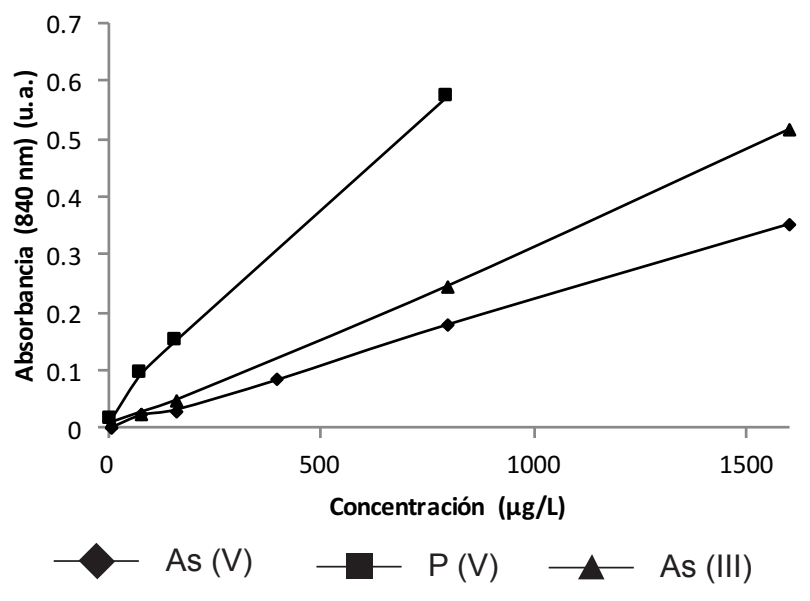

Fig. 4. Curva de calibración para As (III), (V) y P(V) a longitud de onda de $840 \mathrm{~nm}$

las especies químicas en función de la presencia o ausencia de $\mathrm{S}_{2} \mathrm{O}_{4}{ }^{-2}$ para As (V) y mostraron que las diferentes especies químicas de arsenomolibdato y fosfomolibdato podían ser determinadas a $849 \mathrm{~nm}$ y $900 \mathrm{~nm}$ respectivamente. La longitud de onda para la determinación de las especies puede variar en función de los reactivos utilizados en el desarrollo de las especies químicas, como se reporta en este trabajo (Fig. 2).

Hanrahan et al. (2009) desarrollaron un método basado en la técnica del MB acoplado a un sistema de inyección en flujo para la cuantificación de As (V) y (III) en agua. Ellos reportaron 74 y $59 \mu \mathrm{g} / \mathrm{L}$ como LD para As (V) y As (III) respectivamente, con un rango lineal de 75 a $3750 \mu \mathrm{g} / \mathrm{L}$ y valores de 0.9904 a 0.9998 como coeficientes de correlación lineal. La cuantificación en el método actual presenta límites por debajo de lo encontrado por estos autores.

Dhar et al. (2004) informaron sobre la optimización del método colorimétrico MB para medir con precisión las concentraciones de As en el rango de 0.03 a $5.3 \mu \mathrm{mol} / \mathrm{L}$ en aguas subterráneas que contenían de 2 a $30 \mu \mathrm{mol} / \mathrm{L}$ de fosfato disuelto. La optimización incluyó aumentos en las concentraciones de yodato de potasio, ácido ascórbico y tartrato de antimonio uilizados para el pretratamiento de la muestra y el desarrollo del color que redujo significativamente el LD y acortó el tiempo de reacción. La recuperación media del método se realizó mediante el enriquecimiento de muestras de agua subterránea obtenidas de Bangladés con una recuperación del 97 $\pm 5 \%(\mathrm{n}=10)$ así como el intervalo lineal de $0.13 \mathrm{a}$ $13 \mu \mathrm{mol} / \mathrm{L}$ para la cuantificación. Sus parámetros de

CUADRO II. CUANTIFICACIÓN DE AS TOTAL EN AGUA POR MB (MÉTODO PROPUESTO) Y GHAAS (CORRAL 2017). ESTÁNDAR DE CENAM, DMR-312B DE $997 \pm 12 \mathrm{mg} / \mathrm{L}$, DILUIDO A $800 \mu \mathrm{g} / \mathrm{L}$

\begin{tabular}{lllll}
\hline Muestra & \multicolumn{2}{l}{$\begin{array}{l}\text { Arsénico por el } \\
\text { método propuesto }\end{array}$} & \multicolumn{2}{l}{$\begin{array}{l}\text { Arsénico por método } \\
\text { convencional (HGAAS) }\end{array}$} \\
\hline $\begin{array}{l}\text { Agua de } \\
\text { consumo }\end{array}$ & $\mathrm{X} \pm$ d.e. $(\mu \mathrm{g} / \mathrm{L})$ & $\mathrm{DER}$ & $\mathrm{X} \pm$ d.e. $(\mu \mathrm{g} / \mathrm{L})$ & $\mathrm{DER}$ \\
& $71.12 \pm 1.55$ & 0.022 & $62.9 \pm 1.3$ & 0.021 \\
\hline CENAM 800 & $775.71 \pm 9.66$ & 1.246 & $797.9 \pm 7.3$ & 0.925 \\
\hline
\end{tabular}

d.e. desviación estándar 
calidad también se encuentran un orden de magnitud por arriba de lo calculado en el método propuesto.

Otros estudios reportaron concentraciones de As (V) en solución acuosa dentro del orden de $1 \mu \mathrm{g} / \mathrm{L}$ $(0.0001 \mathrm{mg} / \mathrm{L})$; por lo que para utilizar el método del MB es necesaria la preconcentración de As debido a la extremadamente baja absorbancia del heteropoliácido a este nivel de concentración de As (V) (Kiso et al. 2015).

Recientemente, Okazaki et al. (2015) informaron sobre un dispositivo portátil para la determinación de trazas de As en agua subterránea por colorimetría visual equipado con dos accesorios, un cartucho de $\mathrm{CaCO}_{3}$ y un soporte de membrana impregnado con molibdeno; con dicho dispositivo se obtuvo un LD de $5 \mu \mathrm{g} / \mathrm{L}$. La operación del dispositivo fue costosa debido al uso de un cartucho de $\mathrm{CaCO}_{3}$ junto con filtros de membrana. Hasta la fecha, no hay informes disponibles sobre el uso de un procedimiento colorimétrico de varilla para As (V) y la evaluación del rendimiento mediante análisis digital.

\section{CONCLUSIONES}

El método del azul de molibdeno y espectrofotometría de UV-Vis, utilizando L-cisteína y yodato de potasio, permite el análisis de arsénico pentavalente, trivalente y fósforo a nivel de ultra trazas en agua. Con este método se tienen los siguientes parámetros de calidad.

El rango lineal para la detección de As (V) es de 8 a $1600 \mu \mathrm{g} / \mathrm{L}$, la sensibilidad de $0.0002 \mathrm{~L} / \mu \mathrm{g}$ y el LD de $6 \mu \mathrm{g} / \mathrm{L}$. El intervalo lineal para la detección de $\mathrm{P}$ (V) es de 8 a $800 \mu \mathrm{g} / \mathrm{L}$, la sensibilidad de $0.0007 \mathrm{~L} / \mu \mathrm{g}$ y el LD de $2.5 \mu \mathrm{g} / \mathrm{L}$. El rango lineal para la detección de As (III) es de 8 a $1600 \mu \mathrm{g} / \mathrm{L}$, la sensibilidad de $0.0003 \mathrm{~L} / \mu \mathrm{g}$ y el LD de $15.7 \mu \mathrm{g} / \mathrm{L}$.

El método permite realizar análisis por debajo de 10 y $25 \mu \mathrm{g} / \mathrm{L}$ de As total, límites máximos permitidos en agua, que marcan la normativa mundial y mexicana actual, respectivamente.

El gasto operativo está por debajo de los que se tienen con técnicas como la espectrometría de absorción atómica, siendo una opción viable para el análisis de este metaloide.

\section{REFERENCIAS}

Charlot G. (1961). Dosages colorimetriques des elements mineraux. Principes et méthodes. Masson \& Cie, Editores. $10^{\mathrm{a}}$ ed. París, Francia.
Cordero B. E. R. y Cañizares-Macías M. P. (2009). Determination of bioavailable soluble arsenic and phosphates in mine tailings by spectrophotometric Sequential Injection Analysis. Talanta 78(3), 10691076. DOI: 10.1016/j.talanta.2009.01.024.

Corral Escárcega M. E. (2017) Desarrollo de un sensor utilizando membranas poliméricas de inclusión para el análisis de especies tóxicas de As en agua. Tesis para obtener el grado de Maestro en Ciencias en Biotecnología, Facultad de Ciencias Químicas, Universidad Autónoma de Chihuahua, México.

Das J. y Sarkar P. (2016). Environmental Science of arsenate in drinking water. Environmental Science: Water Research \& Technology 2, 693-704.

DOI: $10.1039 / \mathrm{c} 5$ ew00276a.

Dhar R. K., Zheng Y., Rubenstone J. y Van Geen A. (2004). A rapid colorimetric method for measuring arsenic concentrations in groundwater. Analytica Chimica Acta 526(2), 203-209.

DOI: $10.1016 /$ j.aca.2004.09.045

Dhillon A., Nair M. y Kumar D. (2015). Analytical methods for sensing of health-hazardous arsenic from biotic and abiotic natural resources. Analytical Methods 7, 10088-10108. DOI: 10.1039/c5ay02430g.

Hanrahan G., Fan T. K., Kantor M., Clark K., Cardenas S., Guillaume D. W., Khachikian C. S. (2009). Design and development of an automated flow injection instrument for the determination of arsenic species in natural waters. Review of Scientific Instruments 80, 104101. DOI: $10.1063 / 1.3202083$

Kiso Y., Asaoka S., Kamimoto Y., Tanimoto S. y Yokota, K. (2015). Detection tube method for trace level arsenic. Journal of Environmental Chemical Engineering 3(1), 40-45. DOI: 10.1016/j.jece.2014.11.017

Ma, J., Sengupta, M. K., Yuan, D. y Dasgupta, P. K. (2014). Speciation and detection of arsenic in aqueous samples: A review of recent progress in non-atomic spectrometric methods. Analytica Chimica Acta, 831, 1-23. DOI: $10.1016 /$ j.aca.2014.04.029

Martínez L. D. y Gásquez J. A. (2005). Determinación de Arsénico en aguas: diferentes técnicas y metodologías. Sitio Argentino de Producción Animal, (III), 1-6.

Nagul E. A., McKelvie I. D. y Kolev S. D. (2015). The nature of the salt error in the $\mathrm{Sn}$ (II)-reduced molybdenum blue reaction for determination of dissolved reactive phosphorus in saline waters. Analytica Chimica Acta 896, 120-127. DOI: 10.1016/j.aca.2015.07.030.

Nordstrom, D.K. (2002). Worldwide occurrences of arsenic in ground water. Science. 296(5576), 2143-2145. DOI: $10.1126 /$ science.1072375.

Okazaki T., Kuramitz H., Hata N., Taguchi S., Murai K., Okauchi K. y Stetzenbach K. J. (2015). Visual colorimetry for determination of trace arsenic in groundwater 
based on improved molybdenum blue spectrophotometry. Anal. Methods., 7(6), 2794-2799.

DOI: $10.1039 / \mathrm{c} 4 a y 03021 \mathrm{~d}$.

Smedley P.L. y Kinniburgh D.G. (2002). A review of the source, behavior and distribution of arsenic in natural waters. Applied Geochemistry. 17(5), 517-568.

DOI: 10.1016/S0883-2927(02)00018-5.
Tsang S., Phu F., Baum M. M. y Poskrebyshev G. A. (2007). Determination of phosphate/arsenate by a modified molybdenum blue method and reduction of arsenate by $\mathrm{S}_{2} \mathrm{O}_{4}{ }^{2-}$. Talanta 71(4), 1560-1568.

DOI: $10.1016 /$ j.talanta.2006.07.043 